\title{
Network growth for enhanced natural selection
}

\author{
Valmir C. Barbosa, ${ }^{1}$ Raul Donangelo, ${ }^{2,3}$ and Sergio R. Souza ${ }^{2,4}$ \\ ${ }^{1}$ Programa de Engenharia de Sistemas e Computação, COPPE, Universidade Federal do Rio de Janeiro, Caixa Postal 68511, \\ 21941-972 Rio de Janeiro, RJ, Brazil \\ ${ }^{2}$ Instituto de Física, Universidade Federal do Rio de Janeiro, Caixa Postal 68528, 21941-972 Rio de Janeiro, RJ, Brazil \\ ${ }^{3}$ Instituto de Física, Facultad de Ingeniería, Universidad de la República, Julio Herrera y Reissig 565, 11.300 Montevideo, Uruguay \\ ${ }^{4}$ Instituto de Física, Universidade Federal do Rio Grande do Sul, Caixa Postal 15051, 91501-970 Porto Alegre, RS, Brazil
}

(Received 23 December 2008; revised manuscript received 23 March 2009; published 14 August 2009)

\begin{abstract}
Natural selection and random drift are competing phenomena for explaining the evolution of populations. Combining a highly fit mutant with a population structure that improves the odds that the mutation spreads through the whole population tips the balance in favor of natural selection. The probability that the spread occurs, known as the fixation probability, depends heavily on how the population is structured. Certain topologies, albeit highly artificially contrived, have been shown to exist that favor fixation. We present a randomized mechanism for network growth that is loosely inspired in some of these topologies' key properties and demonstrate, through simulations, that it is capable of giving rise to structured populations for which the fixation probability significantly surpasses that of an unstructured population. This discovery provides important support to the notion that natural selection can be enhanced over random drift in naturally occurring population structures.
\end{abstract}

DOI: 10.1103/PhysRevE.80.026115

PACS number(s): 89.75.Fb, 87.23.Kg, 02.10.Ox, 02.50.-r

\section{INTRODUCTION}

Networks of agents that interact with one another underlie several important phenomena, including the spread of epidemics through populations [1], the emergence of cooperation in biological and social systems [2-4], the dynamics of evolution $[5,6]$, and various others $[7,8]$. Typically, the dynamics of such interactions involves the propagation of information through the network as the agents contend to spread their influence and alter the states of other agents. In general, the underlying network structure affects agent behavior greatly and also the global properties that emerge as a consequence of agent interaction. As demonstrated in [6,9-14], subtle structural changes can lead to important variations. The centrality of network structure also occurs in several other areas, such as those surveyed in the chapters of $[15,16]$ and in [17].

In this paper, we focus on the dynamics of evolving populations, particularly on how network structure relates to the ability of a mutation to take over the entire network by spreading from its node of origin. In particular, we target the growth of networks and its possibilities regarding the spread of mutations. The study of network growth can be traced back to the work of Erdös and Rényi on the emergence of the so-called giant component under the steady increase of the network's density of randomly placed edges [18]. In the context of agent networks representing biological or social systems, considerable effort has been dedicated to the study of network growth to account for the appearance of the socalled small-world phenomena [19] and of neighborhoodsize distributions of the power-law type [20].

One of the earliest techniques for the creation of a network in a biological or social domain is that of altering an initial lattice by the removal of randomly selected nodes and connections [21]. Beginning with a lattice has also been shown effective in giving rise to the reduced average dis- tance between nodes that is typical of small-world networks. The key alteration now is the replacement of a randomly selected fraction of the connections, leading in the limit in which all connections are altered to the networks known as random regular graphs [22,23]. Further alterations have also been explored $[24,25]$, resulting in networks with the additional small-world property of tightly clustered neighborhoods.

Other than network creation by alteration of an initial lattice, some prominent methods have been proposed which grow a network by annexing to it one new node at a time, along with connections between the new node and some of the nodes already in the network. The most well-known of these is the so-called method of preferential attachment [20], which selects the nodes for connection to the new node in proportion to a nondecreasing function of how many nodes they are already connected to. The case of a linear function leads to a power-law distribution of neighborhood size [20], but this is lost if the function is nonlinear [26]. Some variations of preferential attachment have appeared that promote the appearance of some desirable properties in real-world networks $[27,28]$ and likewise other methods that succeed in giving rise to similar power laws without resorting to any form of preferential attachment $[29,30]$.

The method we introduce for network growth is rooted in some of the structural properties of networks that are known to influence the probability that a mutation gets spread. We begin with a discussion of these properties and then go on to introduce the method and evaluate it computationally.

\section{FIXATION PROBABILITY}

In evolutionary dynamics, the probability that a mutation occurring at one of a population's individuals eventually spreads through the entire population is known as the mutation's fixation probability, $\rho$. In an otherwise homogeneous 
population, the value of $\rho$ depends on the ratio $r$ of the mutant's fitness to that of the other individuals and it is the interplay between $\rho$ and $r$ that determines the effectiveness of natural selection on the evolution of the population, given its size. In essence, highly correlated $\rho$ and $r$ lead to a prominent role of natural selection in driving evolution; random drift takes primacy, otherwise [31].

Let $P$ be a population of $n$ individuals and, for individual $i$, let $P_{i}$ be any nonempty subset of $P$ that excludes $i$. We consider the evolution of $P$ according to a sequence of steps, each of which first selects $i \in P$ randomly in proportion to $i$ 's fitness, then selects $j \in P_{i}$ randomly in proportion to some weighting function on $P_{i}$, and finally replaces $j$ by an offspring of $i$ having the same fitness as $i$.

When $P$ is a homogeneous population of fitness 1 (except for a randomly chosen mutant, whose fitness is initially set to $r \neq 1$ ), $P_{i}=P \backslash\{i\}$ (where $\backslash$ denotes the set difference), and moreover the weighting function on every $P_{i}$ is a constant (thus choosing $j \in P_{i}$ occurs uniformly at random), this sequence of steps is known as the Moran process [5]. In this setting, evolution can be modeled by a simple discrete-time Markov chain, of states $0,1, \ldots, n$, in which state $s$ indicates the existence of $s$ individuals of fitness $r$, the others $n-s$ having fitness 1 .

In this chain, states 0 and $n$ are absorbing and all others are transient. If $s$ is a transient state, then it is possible either to move from $s$ to $s+1$ or $s-1$, with probabilities $p$ and $q$, respectively, such that $p / q=r$ or to remain at state $s$ with probability $1-p-q$. When $r>1$ (an advantageous mutation), the evolution of the system has a forward bias; when $r<1$ (a disadvantageous mutation), there is a backward bias. And given that the initial state is 1 , the probability that the system eventually reaches state $n$ is precisely the fixation probability, in this case denoted by $\rho_{1}$ and given by

$$
\rho_{1}=\frac{1-1 / r}{1-1 / r^{n}}
$$

(cf. [31]). The probability that the mutation eventually becomes extinct (i.e., that the system eventually reaches state $0)$ is $1-\rho_{1}$. Because $\rho_{1}<1$, extinction is a possibility even for advantageous mutations. Similarly, it is possible for disadvantageous mutations to spread through the entirety of $P$, although in this case the fixation probability decreases exponentially with the size of the population.

In order to consider more complex possibilities for $P_{i}$, we introduce the directed graph $D$ of node set $P$ and edge set containing every ordered pair $(i, j)$ such that $j \in P_{i}$. The case of a completely connected $D$ (in which every node connects out to every other node) corresponds to the Moran process. But in the general case, even though it continues to make sense to set up a discrete-time Markov chain with 0 and $n$ the only absorbing states, analysis becomes infeasible nearly always and $\rho$ must be calculated by computer simulation of the evolutionary steps.

The founding work on this graph-theoretic perspective for the study of $\rho$ is [6], where it is shown that we continue to have $\rho=\rho_{1}$ for a much wider class of graphs. Specifically, the necessary and sufficient condition for $\rho=\rho_{1}$ to hold is that the weighting function be such that, for all nodes, the prob- abilities that result from the incoming weights sum up to 1 (note that this already holds for the outgoing probabilities, thus characterizing a doubly stochastic process for outneighbor selection). In particular, if the weighting function is a constant for all nodes and a node's in-degree (number of in-neighbors) and out-degree (the cardinality of $P_{i}$ for node $i$, its number of out-neighbors) are equal to each other and the same for all nodes, as in the Moran case, then $\rho=\rho_{1}$.

Other interesting structures, such as scale-free graphs [20], are also handled in [6], but the following two observations are especially important to the present study. The first one is that, if $D$ is not strongly connected (i.e., not all nodes are reachable from all others through directed paths), then $\rho>0$ if and only if all nodes are reachable from exactly one of $D$ 's strongly connected components. Furthermore, when this is the case, random drift may be a more important player than natural selection, since fixation depends crucially on whether the mutation arises in that one strongly connected component. If $D$ is strongly connected, then $\rho>0$ necessarily.

The second important observation is that there do exist structures that suppress random drift in favor of natural selection. One of them is the $D$ that in [6] is called a $K$-funnel for $K \geq 2$ an integer. As $n \rightarrow \infty$, the value of $\rho$ for the $K$-funnel approaches the ratio that we denote by $\rho_{K}$, given by

$$
\rho_{K}=\frac{1-1 / r^{K}}{1-1 / r^{K n}} .
$$

Thus, in asymptotic terms the $K$-funnel can be regarded as functionally equivalent to the Moran graph with $r^{K}$ substituting for the fitness $r$. Therefore, the fixation probability can be arbitrarily amplified by choosing $K$ appropriately, provided $r>1$.

Noteworthy additions to the study of [6] can be found in $[32,33]$. In these works, analytical characterizations are obtained for the fixation probability on undirected scale-free graphs, both under the dynamics we have described (in which $j$ inherits $i$ 's fitness) and the converse dynamics (in which it is $i$ that inherits $j$ 's fitness). The main find is that the fixation probability is, respectively for each dynamics, inversely or directly proportional to the degree of the node where the advantageous mutation appears. There have also been analytical characterizations of the fixation probability as relatively parsimonious systems of linear equations, both for directed [34] and some simple undirected [35] graphs.

\section{NETWORK GROWTH}

In this paper, we depart from all previous studies of the fixation probability by considering the question of whether a mechanism exists for $D$ to be grown from some simple initial structure in such a way that, upon reaching a sufficiently large size, a value of $\rho$ can be attained that substantially surpasses the Moran value $\rho_{1}$ for an advantageous mutation. Such a $D$ might lack the sharp amplifying behavior of structures such as the $K$-funnel, but being less artificial might also relate more closely to naturally occurring processes. We respond affirmatively to the question, inspired by the observation discussed above on the strong connectedness of $D$ and 


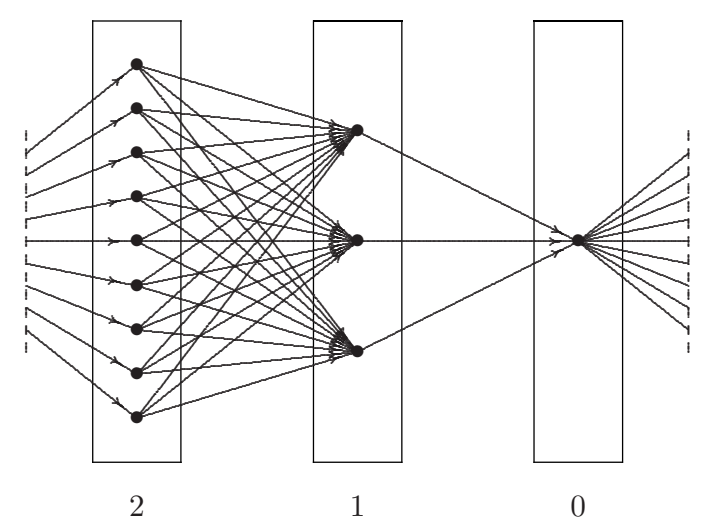

FIG. 1. The 3-funnel for $b=3$. Edges connect back to the nodes in layer 2 from the single node in layer 0 .

using the $K$-funnel as a sieving mechanism to help in looking for promising structures. It should be noted, however, that since other amplifiers exist with capabilities similar to those of the $K$-funnel (e.g., the $K$-superstar [6]), alternatives to the strategy we introduce that are based on them may also be possible.

In a $K$-funnel, nodes are organized into $K$ layers, of which layer $k$ contains $b^{k}$ nodes for some fixed integer $b \geq 2$ and $k=0,1, \ldots, K-1$. It follows that the $K$-funnel has $\left(b^{K}-1\right) /(b-1)$ nodes. A node in layer $k$ connects out to all nodes in layer $k-1$ (modulo $K$, so that an edge exists directed from the single node in layer 0 to each of the $b^{K-1}$ nodes in layer $K-1$ ). A $K$-funnel is then, by construction, strongly connected. An illustration is given in Fig. 1 for $K=b=3$. For a given value of $n$, our strategy for growing $D$ is to make it a layered graph like the $K$-funnel, but letting it generalize on the $K$-funnel by allowing each layer to have any size (number of nodes), provided no layer remains empty.

Graph $D$ is the graph that has $n$ nodes in the sequence $D_{0}, D_{1}, \ldots$ of directed graphs described next. Graph $D_{0}$ has $K$ layers, numbered 0 through $K-1$, each containing one node. The node in layer $k$ connects out to the node in layer $k-1$ (modulo $K$ ). For $t \geq 0$ an integer, $D_{t+1}$ is obtained from $D_{t}$ by adding one new node, call it $i$, to a randomly chosen layer, say layer $k$, according to a criterion to be discussed shortly. Node $i$ is then connected out to all nodes in layer $k-1$ (modulo $K$ ) and all nodes in layer $k+1$ (modulo $K$ ) are connected out to node $i$. Graph $D_{t}$ is then strongly connected for all $t$. The addition of node $i$ to $D_{t}$ is illustrated in Fig. 2. We note that there are as many possibilities for the $D$ that results from the sequence as for partitioning $n$ indistinguishable objects into $K$ nonempty, distinguishable sets arranged circularly, provided we discount for equivalences under rotations of the sets. A lower bound on this number of possibilities is $\left(\begin{array}{l}n \\ K\end{array}\right) / n$, which for $K \ll n$ is roughly $n^{K-1} / K !$.

Before we describe the rule we use to decide which layer is to receive the new node, $i$, it is important to realize that the double stochasticity mentioned earlier implies that $\rho=\rho_{1}$ for $D_{0}$. However, this ceases to hold already for $D_{1}$ and may not happen again as the graph gets expanded. So, whatever the rule is, we are aiming at higher $\rho$ values by giving up on the doubly stochastic character of the process whereby fitness propagates through the graph.

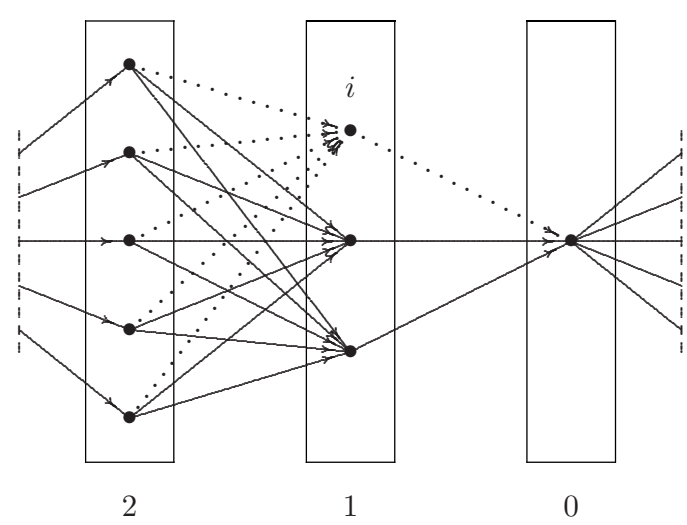

FIG. 2. $D_{t}$ is the graph comprising all nodes but $i$ and all solidline edges. Adding node $i$ to layer 1 and the edges shown as dotted lines yields $D_{t+1}$. Edges connect back to the nodes in layer 2 from the single node in layer 0 .

For $t \geq 0$ and $k$ any layer of $D_{t}$, if we consider the layers in the upstream direction from $k$, we call $k^{+}$the first layer we find whose successor has at most as many nodes as itself. In particular, if the successor of layer $k$ does not have more nodes than $k$, then $k^{+}=k$. Now let $d\left(k^{+}, k\right)$ be the distance from layer $k^{+}$to layer $k$ in $D_{t}$ (i.e., the distance from any node in $k^{+}$to any node in $k$ ).

Layer $k$ is selected to receive node $i$ to yield $D_{t+1}$ with probability

$$
p_{k} \propto\left[K-d\left(k^{+}, k\right)\right]^{a}
$$

for some $a \geq 1$. This criterion is loosely suggested by the topology of the $K$-funnel. It seeks to privilege first the growth of each layer $\ell$ such that $k^{+}=\ell$ for some $k$, then the growth of the layer $k$ that is immediately downstream from $\ell$, provided $k^{+}=\ell$, and so on through the other downstream layers.

\section{COMPUTATIONAL RESULTS AND DISCUSSION}

In our simulations, we use $n \leq 1000$ nearly exclusively and grow a large number of $D$ samples. The calculation of $\rho$ for a given $D$ involves performing several independent simulations (we use 10000 in all cases), each one starting with the fitness- $r$ mutant substituting for any of the $n$ nodes and proceeding as explained earlier until the mutation has either spread through all of $D$ 's nodes or died out (we use constant weighting throughout). The fraction of simulations ending in fixation is taken as the value of $\rho$ for that particular $D$. It is important to note that this method to calculate $\rho$ mimics the evolution of population $P$ rather closely by simulating the evolutionary steps. We then expect the resulting value to approximate the real $\rho$ faithfully, provided sufficiently many independent simulations are conducted. In [6], this has been confirmed to hold for some of the graphs discussed above for which the fixation probability is $\rho_{1}$ and also to hold increasingly well as $n$ grows for some of the graphs whose fixation probability approaches $\rho_{K}$ asymptotically.

This calculation of the fixation probability can be very time-consuming, so we have adopted a mechanism to decide 


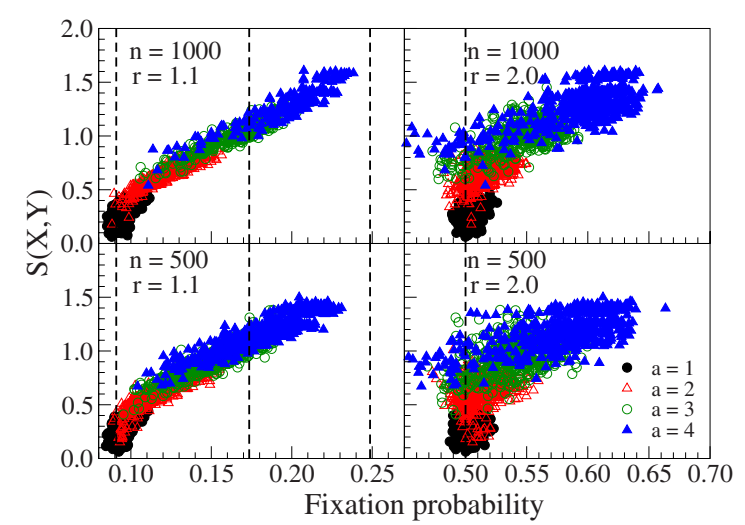

FIG. 3. (Color online) Simulation results for $K=5$. Each graph $D$ for which $C(X, Y)>0.9$ is represented by its fixation probability and by the slope $S(X, Y)$. For each combination of $n$ and $r, 500$ graphs are shown, corresponding roughly to $12 \%$ of the number of graphs that were grown. Dashed lines mark $\rho_{1}$ through $\rho_{3}$ for $r=1.1, \rho_{1}$ for $r=2.0$.

whether to proceed with the calculation for a given $D$ or to discard it. Our mechanism is based on establishing a correlation threshold beyond which $D$ is declared sufficiently similar to the $K$-funnel to merit further investigation. The measure of correlation that we use is the Pearson correlation coefficient between two sequences of the same size, which lies in the interval $[-1,1]$ and indicates how closely the two sequences are to being linearly correlated (a coefficient of 1 means a direct linear dependence). For sequences $X$ and $Y$, the coefficient, denoted by $C(X, Y)$, is given by $C(X, Y)=\operatorname{cov}(X, Y) / \sigma_{X} \sigma_{Y}$, where $\operatorname{cov}(X, Y)$ is the covariance of $X$ and $Y$, and $\sigma_{X}$ and $\sigma_{Y}$ their respective standard deviations.

In our case, $X$ and $Y$ are length- $K$ sequences. If we renumber the layers of $D$ so that the layer with the greatest number of nodes becomes layer $K-1$, the one immediately downstream from it layer $K-2$, and so on through layer 0 , then we let the sequences $X$ and $Y$ be such that $X_{k}=k$ and $Y_{k}=\ln n_{k}$, where $n_{k}$ is the number of nodes in layer $k$. Notice that, when $D$ is the $K$-funnel itself, then $n_{k}=b^{k}$ with $b \geq 2$, whence $Y_{k}=(\ln b) X_{k}$ and $C(X, Y)=1$.

Every $D$ whose sequences $X$ and $Y$ lead $C(X, Y)$ to surpass the correlation threshold is as close to having $n_{k}$ given by some exponential of $k$ as the threshold allows. However, the near-linear dependence of the two sequences is not enough, since the base of such an exponential, which we wish to be as large as possible, can in principle be very small (only slightly above 1), for very gently inclined straight lines. On the other hand, a steeper straight line indicates a faster reduction of layer sizes as we progressively move toward layer 0 from layer $K-1$ through the other layers. In the analysis that follows, then, we also use the slope of the leastsquares linear approximation of $Y$ as a function of $X$, denoted by $S(X, Y)$ and given by $S(X, Y)=\operatorname{cov}(X, Y) / \sigma_{X}^{2}$. For $C(X, Y)$ close to 1 , the base of the aforementioned exponential approaches $e^{S(X, Y)}$.

Our simulation results are summarized in Fig. 3, where $K=5, n=500,1000$, and $r=1.1,2.0$. For each combination and each of four $a$ values $(a=1,2,3,4)$, a scatter plot is

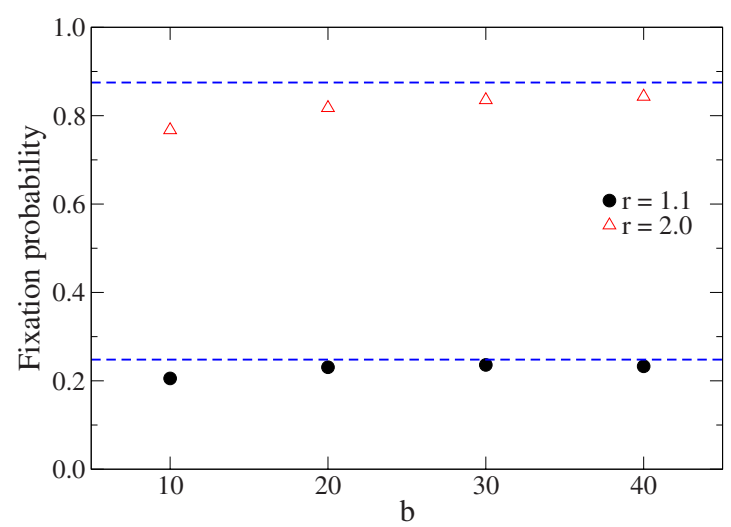

FIG. 4. (Color online) Simulation results for the 3-funnel. Dashed lines mark the values of $\rho_{3}$.

given representing each of the graphs generated by its fixation probability and the slope $S(X, Y)$ for its two sequences, provided $C(X, Y)>0.9$. We see that, in all cases, strengthening the layer-selection criterion by increasing $a$ has the effect of moving most of the resulting graphs away from the Moran probability $\left(\rho_{1}\right)$ and also away from the near- 0 slope.

It is important to notice that, in the absence of the slope indicator for each graph, we would be left with a possibly wide range of fixation probabilities for the same value of $a$, unable to tell the true likeness of the best graphs to the $K$-funnel without examining their structures one by one. In a similar vein, the results shown in Fig. 3 emphasize very strongly the role of our particular choice of a rule for selecting layers, as opposed to merely proceeding uniformly at random. To see this, it suffices that we realize that uniformly random choices correspond to setting $a=0$ in the expression for $p_{k}$ and then we can expect the graphs that pass the correlation threshold to be clustered around the points of $\rho \sim \rho_{1}$ and $S(X, Y) \sim 0$.

We also note a sharp variation in how the fixation probabilities of the graphs relate to the asymptotic fixation probabilities of the $K$-funnel as a mutant's fitness is increased. For $r=1.1$, the graphs exhibiting the highest fixation prob-

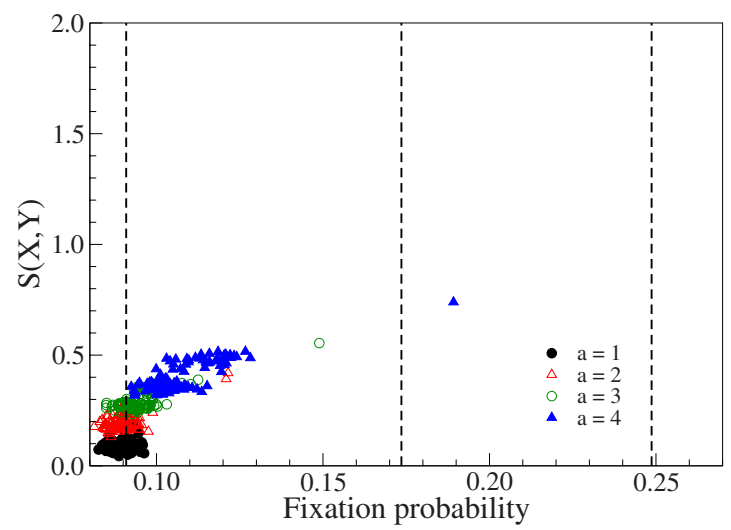

FIG. 5. (Color online) Simulation results for $K=10, n=10000$, and $r=1.1$. Each graph $D$ having $C(X, Y)>0.9$ is represented by its fixation probability and by the slope $S(X, Y)$. There are 100 graphs, corresponding roughly to $0.04 \%$ of the graphs that were grown. Dashed lines mark $\rho_{1}$ through $\rho_{3}$. 
abilities, and also the highest slopes, are such that $\rho$ is somewhere between $\rho_{2}$ and $\rho_{3}$. For $r=2.0$, though, this happens between $\rho_{1}$ and $\rho_{2}(=0.75$, not shown $)$, therefore providing considerably less amplification. Part of the reason why this happens may be simply that the more potent amplifiers are harder to generate by our layer-selection mechanism as $r$ is increased. But it is also important to realize that, even for the $K$-funnel, achieving a fixation probability near $\rho_{K}$ requires progressively larger graphs as $r$ is increased. This is illustrated in Fig. 4 for $K=3$ and the same two values of $r$.

Additional simulation results, for the much larger case of $K=10$ and $n=10000$, are presented in Fig. 5 for $r=1.1$ and $a=1,2,3,4$. Computationally, this case is much more demanding than those of Fig. 3, owing mainly to the number of distinct networks that can occur, as discussed earlier (in fact, for $K=10$ and $n=10000$, this number is at least of the order of $\left.10^{33}\right)$. Consequently, many fewer graphs surpassing the 0.9 correlation threshold were obtained. Even so, one possible reading is that results similar to those reported in Fig. 3 can be expected, but this remains to be seen.
In summary, we have demonstrated that strongly connected layered networks can be grown for which the fixation probability significantly surpasses that of the Moran process. The growth mechanism we use aggregates one new node at a time and chooses the layer to be enlarged by the addition of the new node as a function of how far layers are from those whose populations are the closest upstream local maxima. A great variety of networks can result from this process, but we have shown that correlating each resulting $K$-layer network with the $K$-funnel appropriately works as an effective filter to pinpoint those of distinguished fixation probability. Further work will concentrate on exploring other growth methods and on targeting the growth of more general structures.

\section{ACKNOWLEDGMENTS}

We acknowledge partial support from CNPq, CAPES, FAPERJ BBP grants, the joint PRONEX initiative of CNPq and FAPERJ under Contract No. 26.171.528.2006, and CNPq-PROSUL.
[1] M. Barthélemy, A. Barrat, R. Pastor-Satorras, and A. Vespignani, Phys. Rev. Lett. 92, 178701 (2004).

[2] F. C. Santos and J. M. Pacheco, Phys. Rev. Lett. 95, 098104 (2005).

[3] H. Ohtsuki, C. Hauert, E. Lieberman, and M. A. Nowak, Nature (London) 441, 502 (2006).

[4] P. D. Taylor, T. Day, and G. Wild, Nature (London) 447, 469 (2007).

[5] P. A. P. Moran, Proc. Cambridge Philos. Soc. 54, 60 (1958).

[6] E. Lieberman, C. Hauert, and M. A. Nowak, Nature (London) 433, 312 (2005).

[7] A. Grönlund and P. Holme, Adv. Complex Syst. 8, 261 (2005).

[8] G. Szabó and G. Fáth, Phys. Rep. 446, 97 (2007).

[9] H. Ohtsuki, M. A. Nowak, and J. M. Pacheco, Phys. Rev. Lett. 98, 108106 (2007).

[10] H. Ohtsuki, J. M. Pacheco, and M. A. Nowak, J. Theor. Biol. 246, 681 (2007).

[11] Z.-X. Wu, X.-J. Xu, and Y.-H. Wang, Chin. Phys. Lett. 23, 531 (2006).

[12] J.-Y. Guan, Z.-X. Wu, Z.-G. Huang, X.-J. Xu, and Y.-H. Wang, Europhys. Lett. 76, 1214 (2006).

[13] B. J. Kim, A. Trusina, P. Holme, P. Minnhagen, J. S. Chung, and M. Y. Choi, Phys. Rev. E 66, 021907 (2002).

[14] A. Szolnoki and G. Szabó, Europhys. Lett. 77, 30004 (2007).

[15] Handbook of Graphs and Networks, edited by S. Bornholdt and H. G. Schuster (Wiley-VCH, Weinheim, Germany, 2003).

[16] The Structure and Dynamics of Networks, edited by M. Newman, A.-L. Barabási, and D. J. Watts (Princeton University Press, Princeton, NJ, 2006).

[17] S. Boccaletti, V. Latora, Y. Moreno, M. Chavez, and D.-U. Hwang, Phys. Rep. 424, 175 (2006).

[18] P. Erdős and A. Rényi, Magyar Tud. Akad. Mat. Kutat. Int. Kyzl. 5, 17 (1960).
[19] S. Milgram, Psychol. Today 2, 60 (1967).

[20] A.-L. Barabási and R. Albert, Science 286, 509 (1999).

[21] M. A. Nowak, S. Bonhoeffer, and R. M. May, Int. J. Bifurcation Chaos Appl. Sci. Eng. 4, 33 (1994).

[22] N. C. Wormald, in Surveys in Combinatorics, edited by J. D. Lamb and D. A. Preece, London Mathematical Society Lecture Notes Series Vol. 267 (Cambridge University Press, Cambridge, England, 1999), pp. 239-298.

[23] N. C. Wormald, J. Comb. Theory, Ser. B 31, 168 (1981).

[24] D. J. Watts and S. H. Strogatz, Nature (London) 393, 440 (1998).

[25] M. E. J. Newman and D. J. Watts, Phys. Rev. E 60, 7332 (1999).

[26] P. L. Krapivsky, S. Redner, and F. Leyvraz, Phys. Rev. Lett. 85, 4629 (2000).

[27] S. N. Dorogovtsev, J. F. F. Mendes, and A. N. Samukhin, Phys. Rev. E 63, 062101 (2001).

[28] L. N. A. Amaral, A. Scala, M. Barthélemy, and H. E. Stanley, Proc. Natl. Acad. Sci. U.S.A. 97, 11149 (2000).

[29] V. C. Barbosa, R. Donangelo, and S. R. Souza, Phys. Rev. E 74, 016113 (2006).

[30] V. C. Barbosa, R. Donangelo, and S. R. Souza, Physica A 387, 1016 (2008).

[31] M. A. Nowak, Evolutionary Dynamics (Harvard University Press, Cambridge, MA, 2006).

[32] T. Antal, S. Redner, and V. Sood, Phys. Rev. Lett. 96, 188104 (2006)

[33] V. Sood, T. Antal, and S. Redner, Phys. Rev. E 77, 041121 (2008).

[34] N. Masuda and H. Ohtsuki, New J. Phys. 11, 033012 (2009).

[35] M. Broom and J. Rychtár, Proc. R. Soc. London, Ser. A 464, 2609 (2008). 